\title{
Polybrominated diphenyl ether quinone exposure induces atherosclerosis progression via CD36-mediated lipid accumulation, NLRP3 inflammasome activation and pyroptosis
}

Yuting Wang ${ }^{1}$, Changyu Fang ${ }^{1}$, Lei Xu ${ }^{1}$, Bingwei Yang ${ }^{1}$, Erqun Song ${ }^{1}$, Yang Song ${ }^{1,2, *}$

1. Key Laboratory of Luminescence Analysis and Molecular Sensing, Ministry of Education, College of Pharmaceutical Sciences, Southwest University, 2 Tiansheng Rd, Beibei District, Chongqing, 400715, China;

2. State Key Laboratory of Environmental Chemistry and Ecotoxicology, Research Center for EcoEnvironmental Sciences, Chinese Academy of Sciences, 18 Shuangqing Rd, Haidian District, Beijing, 100085, China

${ }^{*}$ Corresponding Author: State Key Laboratory of Environmental Chemistry and Ecotoxicology, Research Center for Eco-Environmental Sciences, Chinese Academy of Sciences, 18 Shuangqing Rd, Haidian District, Beijing, 100085, China; E-mail: yangsong@rcees.ac.cn 
Table of Content:

Supplementary Fig. 1. PBDEQ promoted lipid accumulation mainly depended on CD36 in J774A.1 cells.

Supplementary Fig. 2. PBDEQ induced pyroptosis in Ox-LDL-treated J774A.1 cells via CD36. 
A

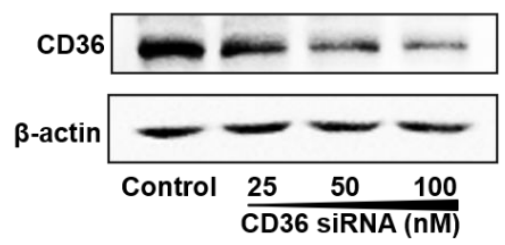

D

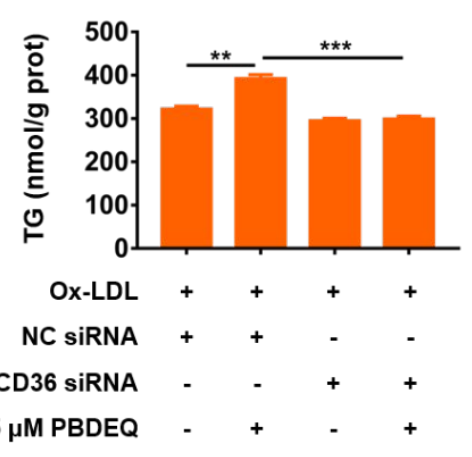

G

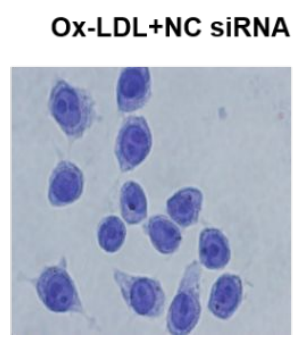

B

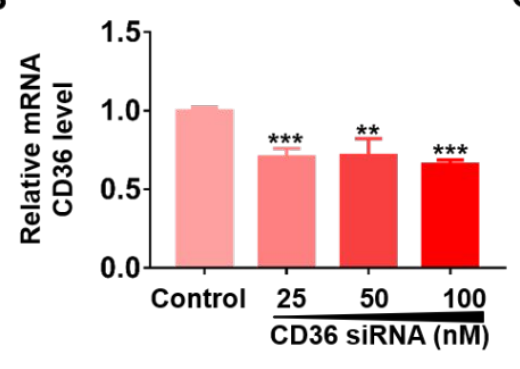

E

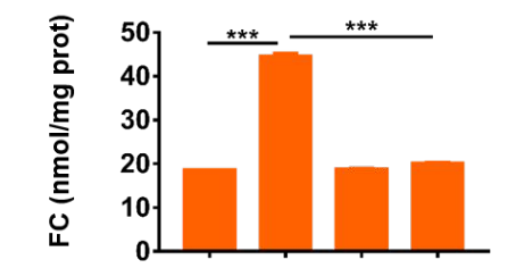

C

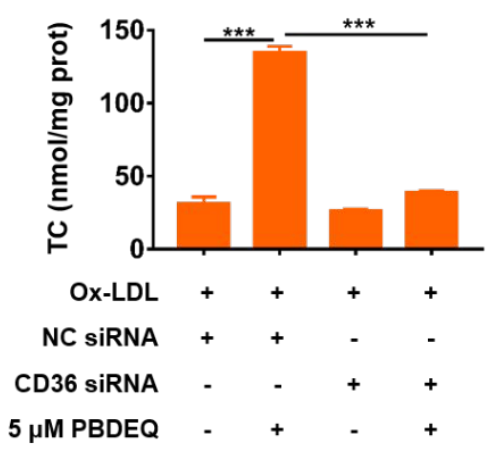

$\mathbf{F}$
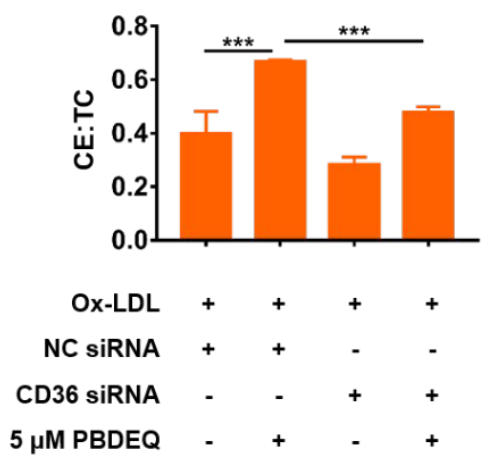

Supplementary Fig. 1 PBDEQ promoted lipid accumulation mainly depended on CD36 in J774A.1 cells. cells were transfected with $50 \mathrm{nM}$ scramble siRNA as control, $25 \mathrm{nM}, 50 \mathrm{nM}$ and $100 \mathrm{nM} \mathrm{CD} 36$ siRNA for $48 \mathrm{~h}$. (A) and (B) CD36 siRNA silenced the expression of CD36 at both protein and mRNA levels. After transfected with 50 nM NC siRNA or CD36 siRNA for 36 h, J774A. 1 cells were exposed to $10 \mu \mathrm{g} / \mathrm{mL}$ Ox-LDL and $5 \mu \mathrm{M}$ PBDEQ for $12 \mathrm{~h}$. (C) TC, (D) TG and (E) FC contents were measured by assay kit. (F) the ratio of $\mathrm{CE}$ to TC. (G) Oil red O staining was utilized to detected the effect of CD36 on PBDEQ induced cellular lipid accumulation (scale bar: $10 \mu \mathrm{m}$ ). Data were presented as the mean \pm SD of at least three independent experiments. ${ }^{*} \mathrm{p}<0.05,{ }^{*} \mathrm{p}<0.01$, and ${ }^{* * *} \mathrm{p}<0.001$ compared to control. 
A

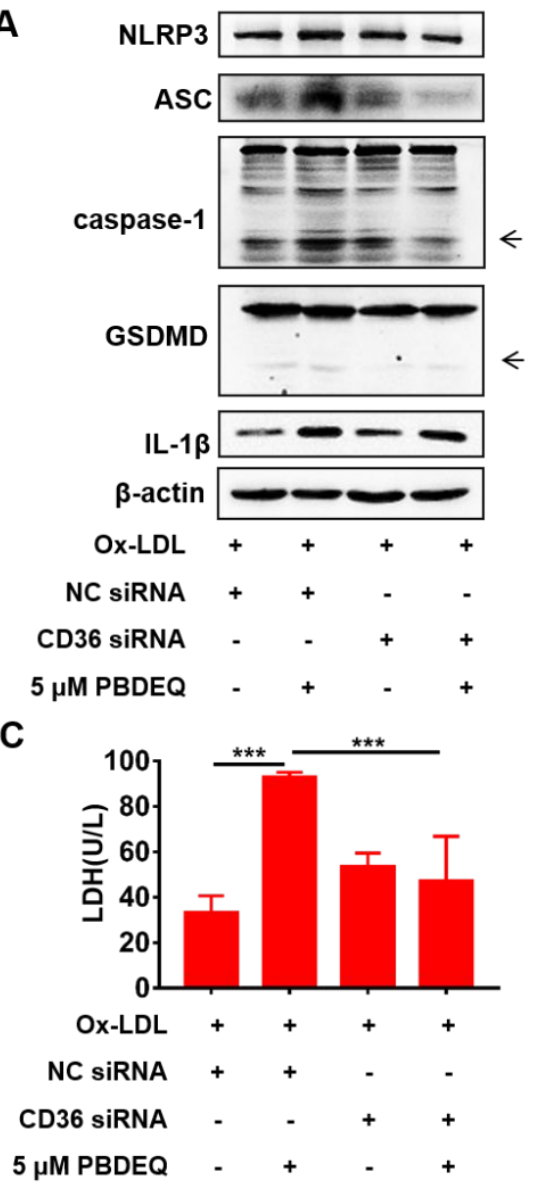

B

Ox-LDL+NC siRNA Ox-LDL+NC siRNA+5 $\mu$ M PBDEQ

Ox-LDL+CD36 siRNA Ox-LDL+CD36 siRNA+5 $\mu$ M PBDEQ
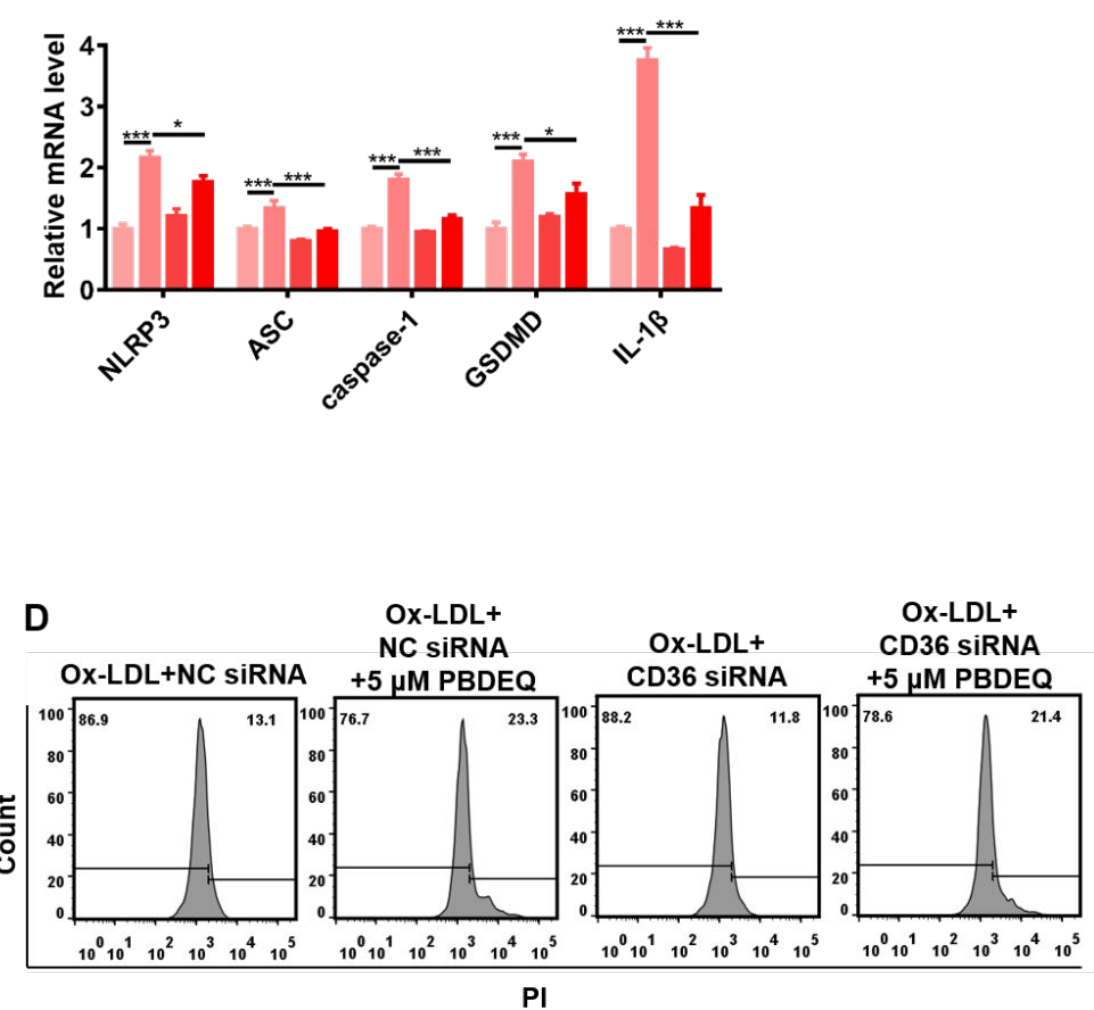

Supplementary Fig.2 PBDEQ induced pyroptosis in Ox-LDL-treated J774A.1 cells via CD36. J774A.1

cells were transfected with $50 \mathrm{nM} \mathrm{NC}$ siRNA or CD36 siRNA for $36 \mathrm{~h}$, then treated with $10 \mu \mathrm{g} / \mathrm{mL}$ Ox-LDL and $5 \mu \mathrm{M}$ PBDEQ. (A) Silence of CD36 inhibited the protein expression of NLRP3, GSDMD, caspase-1, ASC and IL-1 $\beta$ expression. (B) RT-PCR analyzed the mRNA levels of NLRP3, GSDMD, caspase-1, ASC and IL-1 $\beta$. (C) and (D) Silence of CD36 decreased LDH and PI levels. Data were presented as the mean $\pm \mathrm{SD}$ of at least three independent experiments. ${ }^{*} \mathrm{p}<0.05,{ }^{* *} \mathrm{p}<0.01$, and ${ }^{* * *} \mathrm{p}$ $<0.001$ compared to control. 